\title{
Raman microspectroscopy for species identification and mapping within bacterial biofilms
}

\author{
Brooke D Beier ${ }^{1}$, Robert G Quivey ${ }^{2}$ and Andrew J Berger ${ }^{1 *}$
}

\begin{abstract}
A new method of mapping multiple species of oral bacteria in intact biofilms has been developed, using the optical technique of confocal Raman microscopy. A species classification algorithm, developed on dried biofilms, was used to analyze spectra of hydrated biofilms containing two microbial species central to dental health: Streptococcus sanguinis and Streptococcus mutans. The algorithm transferred successfully to the hydrated environment, correctly identifying the species of origin of single-species biofilms. We then used the algorithm successfully both to detect the presence of two species in mixed biofilms and to create spatial maps within these biofilms.
\end{abstract}

Keywords: Raman spectroscopy, Confocal microscopy, Biofilms, Bacteria, Dental plaque

\section{Introduction}

The identification of microbial species within sample specimens is relevant to both the microbiological research laboratory and the clinical setting. A number of standard methods of species identification are currently used. Selective plating may be used to identify the constituent species and to obtain an order-of-magnitude estimate of the initial concentrations of bacteria present in a sample. Since this method involves serial dilutions, it can take up to several days and is insensitive to cells that are dead or incapable of reproduction by the time the sample is procured. Quantitative polymerase chain reaction (qPCR) is highly sensitive to the initial concentrations of known species, but it requires prior knowledge of their genomes in order to provide specificity. Both qPCR and selective plating disrupt the initial sample's architecture, and are thus ill-suited for spatial mapping studies of specimens such as biofilms. Fluorescence in situ hybridization (FISH) can provide spatial resolution, but requires sample fixation (eliminating the potential to study a sample over time), extensive preparation steps, and a geneticallytargeted exogenous marker. A new measurement method that could map the spatial distribution of multiple species

\footnotetext{
*Correspondence: ajberger@optics.rochester.edu

1 The Institute of Optics, University of Rochester, 275 Hutchison Rd., Rochester, NY 14627, USA

Full list of author information is available at the end of the article
}

in intact, unfixed specimens in a label-free, non-contact manner would therefore be valuable. Such a technique could also make longitudinal study of samples like biofilms a possibility.

Optical spectroscopy offers the ability to acquire chemically-specific information at micron-scale resolution without sample contact. One such method is Raman spectroscopy, which has been used extensively for biological applications. Raman spectroscopy detects the presence of molecular bonds via the inelastic scattering of laser light; each molecule imparts a different "fingerprint pattern" on the spectrum of the scattered light. This provides specificity to subtle biochemical differences between samples, useful in discrimination of cell types or monitoring the progression of disease. Raman spectroscopy has been used to study biomedical specimens including tissues, biofluids, and bacterial cells (Maquelin et al. 2002; Rösch et al. 2005; Willemse-Erix et al. 2009); extensive reviews are provided by Hanlon et al. (2000) and by Ellis and Goodacre (2006).

In the work described here, Raman spectroscopy implemented through a confocal microscope has been used to distinguish between two species of streptococci, Streptococcus sanguinis and S. mutans, grown in biofilm form. These bacteria are of particular interest due to their relationship with oral health, being the two most populous

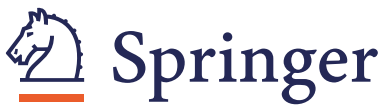

(c) 2012 Beier et al; licensee Springer. This is an Open Access article distributed under the terms of the Creative Commons Attribution License (http://creativecommons.org/licenses/by/2.0), which permits unrestricted use, distribution, and reproduction in any medium, provided the original work is properly cited. 
species present within dental plaque (Socransky and Manganiello 1971). Streptococcus mutans has been identified as the most cariogenic species in plaque, with elevated levels being linked to increased risk of tooth decay (Loesche 1986; Marsh 1999). Streptococcus sanguinis is associated with being the primary component of healthy plaque (Socransky and Manganiello 1971). Examining the relative balance between these two species can provide an indication of a patient's risk of tooth decay.

Previous work by our group led to the successful identification of the species of bacteria present in single-species biofilms that had been dehydrated before analysis (Beier et al. 2010). In that study, biofilm samples had been transferred from their original substrates, much like a plaque scraping might be harvested from a tooth surface. Dehydrated samples were used for training the species identification model because sample volumes would contain denser cellular content leading to higher signal levels. Since only single-species biofilms were used in the construction of the training set, we chose this enhancement in signal levels over the preservation of structural information. Here, we apply this model to map species distributions within simple hydrated biofilms. Confirmation of the model's successful transfer is performed using intact single-species biofilms. With the ability to identify the species within hydrated biofilms in situ, the model is used to perform species identifications of volumes within a two-species mixed biofilm.

\section{Materials and methods Biofilm preparations}

Streptococcus mutans ATCC strain UA159 and S. sanguinis ATCC strain 10904 were examined in these studies. Although the two species have different preferred growth media, identical preparations were used when preparing pure biofilms of each species. The same nutrients were also used when creating two-species biofilms. This was done to ensure that the chemical differences detected by Raman spectroscopy were in fact indicators of differences in the species' biofilms and not an artifact from differences in the chemical content of the nutrients used.

\section{Single-species biofilms}

Cells were taken from stocks stored at $-80^{\circ} \mathrm{C}$ and streaked onto agar plates containing brain heart infusion medium (BD Difco, Franklin Lakes, New Jersey). After $24 \mathrm{hr}$, a few colonies were selected and used to inoculate a liquid culture containing $10 \mathrm{~mL}$ of Todd Hewitt (TH) broth (VWR International, West Chester, Pennsylvania) with $0.5 \%(\mathrm{w} / \mathrm{v})$ sucrose. The presence of sucrose allows the bacteria in suspension to begin secretion of extracellular polysaccharides (EPS) that are vital for the formation of a biofilm structure. After another $24 \mathrm{hr}, 1 \mathrm{~mL}$ of the resuspended liquid culture was added to $49 \mathrm{~mL}$ of $\mathrm{TH}$ broth with $0.5 \%$ sucrose. At this point, a microscope slide was introduced into the culture to serve as a substrate for the biofilm. The biofilm slide was moved to fresh media every $24 \mathrm{hr}$ until a total of 4 days of growth on the substrate had been reached. In the calibration set used previously to develop our species prediction model, the sugar had been changed for the last three days to $0.5 \%$ glucose to produce more cell-rich biofilms with suppressed levels of EPS. For the sample set consisting of intact biofilms presented here, however, the sugar source was maintained as $0.5 \%$ sucrose throughout to encourage the biofilms to be more robust and space-filling, as we could no longer rely upon dehydration-induced sample compaction to increase the sample density and thus the Raman signal strength. The difference in EPS levels between the two data sets was thus a potential problem for successful calibration transfer.

For the biofilms in the calibration set, ordinary glass microscope slides were used since each biofilm was ultimately transferred from the substrate before Raman analysis, much like a plaque scraping might be transferred. For the hydrated biofilms of interest in these studies (examined in situ), quartz microscope slides were chosen in order to avoid the high levels of fluorescence associated with using NIR illumination on a glass substrate.

\section{Two-species biofilms}

Previous work by our group included the creation of artificially mixed, two-species biofilms where pure biofilms of each species were manually combined in a controlled way immediately before study, in order to investigate the spatial capabilities of our system. In that study, correct identifications of species were reliably made for locations as close as $2 \mu \mathrm{m}$ to a boundary between species (Beier et al. 2010). It is of course more biologically relevant to consider multispecies biofilms grown from a common culture. In the studies described here, two-species biofilms of S. sanguinis and S. mutans were grown by first initiating a biofilm of $S$. sanguinis for 3 days before introducing liquid culture of $S$. mutans. This time delay was necessary because for the batch growth conditions used throughout these studies, S. mutans would quickly dominate over $S$. sanguinis due to $S$. mutans 's proclivity for thriving in acidic environments. It was found that the delayed introduction of S. mutans followed by $12 \mathrm{hr}$ of coexistence led to a biofilm that contained sufficient amounts of both species. Similar sample preparations consisting of 4 days of $S$. sanguinis and $3 \mathrm{hr}$ coexistence with $S$. mutans or 2 days of $S$. sanguinis and 3 days of coexistence with S. mutans led to biofilms that were indistinguishable from pure biofilms of S. sanguinis and S. mutans, respectively.

\section{System design}

Raman spectroscopy was performed using a homebuilt confocal Raman microspectroscopy system described 
previously (Beier and Berger 2009) and shown schematically in Figure 1. An $830 \mathrm{~nm}$ diode laser (Innovative Photonic Solutions, Monmouth Junction, New Jersey) was used as the excitation source for Raman scattering. A near-IR wavelength was chosen in order to prevent thermal effects on samples studied in situ. This wavelength also offered the advantage of avoiding fluorescence that is commonly observed in biological materials. The laser was directed through a spectral bandpass filter (Chroma Technology Corp., Bellows Falls, Vermont) and a spatial filter (10x objective, Newport Corp., Irvine, California; $10 \mu \mathrm{m}$ pinhole). The beam was reflected from a notch filter (Semrock, Inc., Rochester, New York) at near-normal incidence before being directed into the upright microscope (Eclipse E400, Nikon Instruments Inc., Melville, New York). The beam was then focused by a $60 \mathrm{x}$, 1.0 numerical aperture (NA) water immersion objective (Nikon Corp., Tokyo, Japan). The focal spot at the sample plane was $\sim 1.5 \mu \mathrm{m}$ in diameter, delivering $\sim 40 \mathrm{~mW}$ of laser power to the sample. Epidirected Raman scattered light (Stokes-shifted in wavelength) was then collected by the same objective and directed to pass through the notch filter before being focused onto the $100 \mu \mathrm{m}$ core of a multimode optical fiber, which served as a confocal pinhole. The fiber guided the light to a spectrometer (HoloSpec f/1.8, Kaiser Optical
Systems Inc., Ann Arbor, Michigan) connected to a thermoelectrically cooled, front-illuminated, open electrode charge-coupled device (CCD) array (DU420-OE, Andor Technology PLC, Belfast, Northern Ireland) that was used to record the spectra. The CCD and stage were controlled using code written in-house within MATLAB $\mathbb{B}$ (Version 7.8.0, The MathWorks TM, Inc., Natick, Massachusetts).

The system has a spectral resolution of $\sim 7 \mathrm{~cm}^{-1}$, as measured from neon gas emission lines. The axial sectioning depth is $\sim 7 \mu \mathrm{m}$, as determined from the derivative of the response curve when scanning into plastic, following the method described by Caspers et al. (2000). Combining the axial sectioning depth with the focal spot diameter of $\sim 1.5 \mu \mathrm{m}$ gives a confocal volume, or voxel size, of $\sim 8 \mu \mathrm{m}^{3}$ to be probed in each location. Scanning of the samples was achieved through the use of an electronicallycontrolled stage ( $\mathrm{x}-\mathrm{y}$ : Applied Scientific Instrumentation, Eugene, Oregon; z: Nikon).

Microscope slides fully coated by biofilm material were loaded with enough water to maintain water-immersion at the microscope objective for many hours. Sufficient air space was provided underneath the slide to drain away water seepage and prevent motion artifacts. In situ study of these samples meant that the structure of the biofilms, including species distributions in two-species biofilms, was intact and available for study. Scanning was

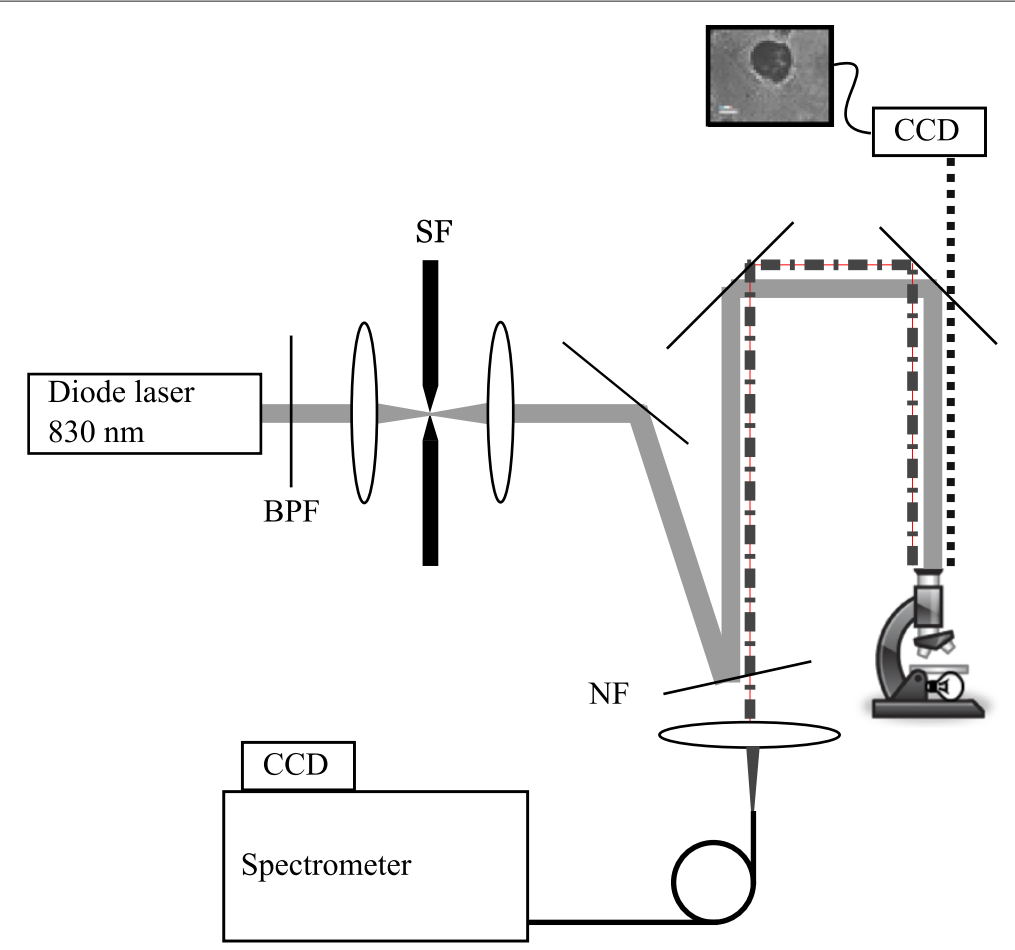

Figure 1 Confocal Raman microscope. Schematic overview of confocal Raman microscope; see text for details. Abbreviations: BPF, bandpass filter; SF, spatial filter; NF, notch filter. 
performed to create maps of slices perpendicular to the substrate (XZ scans) since the structures in this orientation would be more biologically interesting, in terms of the arrangement of bacteria in relation to the substrate.

\section{Data acquisition and processing}

Spectra were acquired for six frames of $30 \mathrm{~s}$ per voxel (i.e. confocal volume). The spectra were then subjected to preprocessing including cosmic-ray and system background removal, as well as spectral throughput correction. Fluorescent background was removed using a modified polynomial-fitting method described previously (Beier and Berger 2009), making use of the photobleaching lineshape between successive frames as a background fitting parameter. After the frames were preprocessed individually, they were averaged to give one spectrum per voxel. Due to a slight shift in the laser's excitation wavelength from that used for the training set, all spectra were then recalibrated to align the $1003 \mathrm{~cm}^{-1}$ phenylalanine peak (Wagner et al. 2009) and resampled to a common wavenumber axis. For further analysis, only data from the wavenumber region of 706 to $1810 \mathrm{~cm}^{-1}$ was retained for each spectrum.

Some voxels were found to have insufficient signal levels for reliable species prediction, likely due to low cellular content in a given voxel or insufficient light penetration into deeper regions. An initial screening step was performed to reject such voxels from further consideration.

After the spectral data was preprocessed, it was submitted to the species identification model described previously (Beier et al. 2010). Briefly, principal component (PC) analysis was used for data compression and noise reduction before submitting the PC scores to logistic regression for species prediction.

\section{Results}

As mentioned above, the species prediction model was originally constructed from dehydrated biofilm samples and was here applied to the study of hydrated biofilms, in situ. For the transfer of this model to a new sample preparation, a test set of single-species biofilms was first examined. The mean spectra of the newly-measured $S$. sanguinis and $S$. mutans biofilms, shown in Figure 2, appear nearly identical. In such cases, ad hoc classification models based upon a few visibly different peaks (e.g. near 920 and $1100 \mathrm{~cm}^{-1}$, marked with (*') perform poorly at the single-spectrum level, where noise is higher. Figure 2 emphasizes the importance of using a multivariate model (the PC-based technique referenced in the previous section) to utilize information throughout the full spectrum in constructing the classification formula.

Table 1 shows the species identification results obtained for the new test set of 352 spectra from regions within 10 different single-species biofilms. The diagonal entries

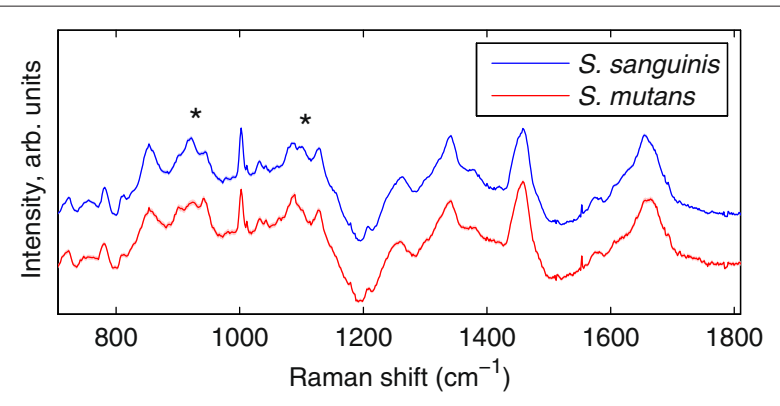

Figure 2 Mean Raman spectra. Mean Raman spectra for S. sanguinis and S. mutans from the validation set of hydrated single-species biofilms. While certain spectral regions are visibly different (marked with $\left.{ }^{* \prime}\right)$, multivariate methods using the full spectra were used for species classification.

in this confusion matrix represent Raman-based species assignments that agree with the (known) species from which the biofilm was prepared, while off-diagonals represent errors. $92 \%$ of all voxels of S. sanguinis and $94 \%$ of S. mutans were properly identified based on their Raman spectra. Considering all voxels from either species, 93\% were properly identified. This study therefore established the baseline error rate in classification to be around $7 \%$.

After confirming the successful transfer of the original model to working with hydrated samples, further studies examined biofilms from mixed culture. A total of five mixed biofilms were studied, each at a number of positions both laterally and in depth. Figure 3 plots the percentage of voxels that were classified as $S$. mutans in each biofilm. As the figure shows, four of the five mixed-species biofilms had Raman-assigned minority-species levels that significantly exceeded the $7 \%$ baseline error rate (Student's t-test, 95\% confidence). The spatial organization of species assignments was also examined. Figure 4 plots the Ramanbased species assignments in a depth-slice through one of the mixed-species biofilms. As can be seen, the species assignments tended to be clustered into contiguous spatial regions as opposed to being evenly distributed. Such assignments are plausible, given the nature of cell proliferation, although there is no reference method currently available to provide confirmation.

Table 1 Species identification performance

\begin{tabular}{lcc}
\hline & \multicolumn{2}{c}{ Known species } \\
\cline { 2 - 3 } & S. sanguinis & S. mutans \\
\hline Predicted & 236 & 6 \\
S. sanguinis & 20 & 90 \\
S. mutans & & 90 \\
\hline
\end{tabular}

Confusion matrix summarizing the performance of the species identification model on hydrated, intact biofilms. The columns indicate the known/true species, while the rows indicate the prevalence of experimentally predicted species in reference to the known species. 


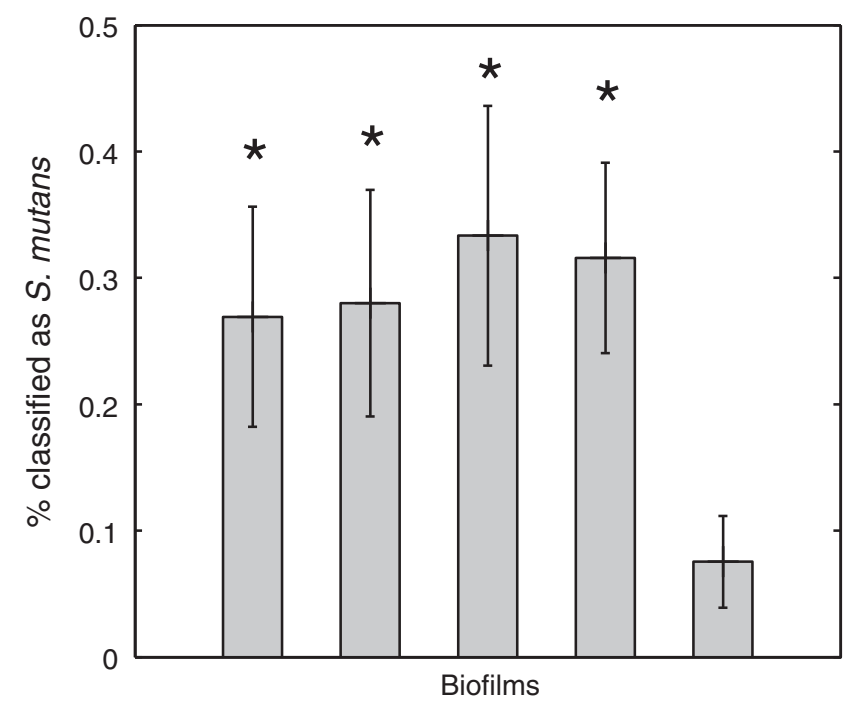

Figure 3 Species predictions. Species prediction results for 5 two-species mixed biofilms. A normal approximation to the binomial distribution has been used to determine the standard error in the mean. Biofilms with proportions of S. mutans statistically significantly different from results seen in pure S. sanguinis biofilms are marked by ${ }^{\prime * \prime}$.

\section{Discussion}

Examination of the test set of hydrated biofilm samples showed that overall, $93 \%$ of voxels were properly identified using the transferred model. Our previous work saw $96 \%$ correct identification of species (Beier et al. 2010). The move to the new sample type involved several changes relative to the original training set, including a replacement of the laser with a corresponding $2 \mathrm{~nm}$ wavelength difference in excitation, the use of a water-immersion objective rather than air-immersion, the addition of depth scanning,

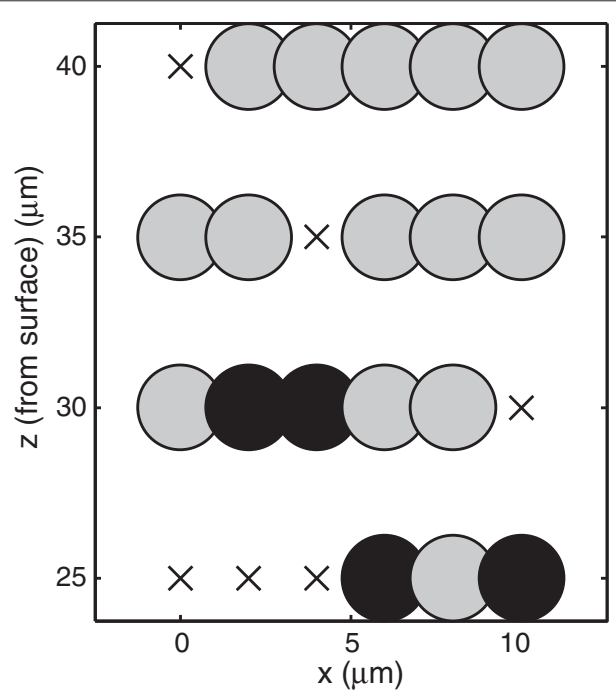

Figure 4 Scan through a mixed biofilm. XZ scan through a mixed biofilm. Gray: S. sanguinis; black: S. mutans; ' $x$ ': insufficient signal. Although S. mutans was introduced later, locations classified as S. mutans were closer to the substrate. and a decrease in signal level associated with decreased concentrations within hydrated biofilms. The high performance on this single species confirmation set given these system changes indicates the robustness of the prediction model.

With the ability to properly identify the species within hydrated samples thus confirmed, biofilms from a mixed culture were analyzed. As mentioned above, the spatial arrangement of clusters of like-classification was a reasonable result, though it was not directly verifiable. What can be asserted with confidence, however, is that in mixedspecies biofilms the "minority" species was typically assigned frequently enough to exceed the baseline error rate of $7 \%$ seen in single-species studies. The fact that four of the five mixed-species biofilms had Raman-assigned minority-species levels that significantly exceeded 7\% (Figure 3) indicates that the presence of both species has been detected and quantified in the mixed biofilm samples. The fifth biofilm, which did not see a statistically significant level of the minority species, may simply have been scanned in a region occupied by a single species.

There is another interesting element to the depth scan in Figure 4. Although S. mutans was introduced days after S. sanguinis, the locations classified as $S$. mutans tended to be closer to the substrate, as if the $S$. mutans had migrated beneath the layer of S. sanguinis. This observation was consistent across all four of the biofilms in which significant levels of both species were observed. For the particular species in this study, this could potentially have significance as it relates to the cariogenic properties of dental plaque. Further experiments are needed to explore this initial observation. 
In summary, Raman spectroscopy has been implemented through a confocal microscope and used to successfully classify oral bacteria in hydrated biofilms of one or two species. A model constructed with spectra from dehydrated single-species biofilms has been transferred for the species prediction of hydrated samples. In a test set of single-species hydrated biofilms, voxel-by-voxel species assignments were $93 \%$ accurate.

When two-species biofilms from mixed culture were examined, the presences of both $S$. sanguinis and S. mutans were detected in four out of five biofilm regions. We cannot rule out the possibility that the remaining biofilm was simply scanned over a single-species region. The spatial arrangement of species observed in these mixed biofilms has potential implications for the study of dental plaque cariogenicity, though this aspect of our study would require further investigation. To the best of our knowledge, this is the first time two bacterial species of the same genus and sub-genus group have been mapped in a biofilm using Raman spectroscopy.

While the specific experiments described above have shown the discrimination between $S$. sanguinis and $S$. mutans, the method presented here could be applied to the study of other microbes. Although the formation of Raman-based species maps is not fast, the technique offers the ability to use intrinsic chemical differences between cells to create multidimensional maps of microbial structures without extensive knowledge of the cells' genomes and without requiring any invasive sample preparation that could potentially alter the sample under study.

\section{Competing interests}

The authors declare that they have no competing interests.

\section{Acknowledgements \\ The authors would like to acknowledge Roberta Faustoferri and Ben Metcalf from the Center for Oral Biology for advice in microbiological preparations. Helpful conversations with Hyun Koo and Jin Xiao are gratefully acknowledged. This work was partially funded through National Institutes of Health (NIH) Grants 1-R21-DE016111-01A1 (AJB), DE0-17157 (RGQ), and DE0-13683 (RGQ), and by graduate fellowships from the Department of Defense's (DoD's) National Defense Science \& Engineering (NDSEG) program and the National Science Foundation's Graduate Research Fellowship Program (NSF GRFP) (BDB).}

\section{Author details}

${ }^{1}$ The Institute of Optics, University of Rochester, 275 Hutchison Rd., Rochester, NY 14627, USA. ${ }^{2}$ Center for Oral Biology, University of Rochester Medical Center, 601 Elmwood Ave., Rochester, NY 14642, USA.

Received: 29 May 2012 Accepted: 13 July 2012

Published: 13 July 2012

\section{References}

Beier BD, Berger AJ (2009) Method for automated background subtraction from raman spectra containing known contaminants. Analyst 134(6): 1198-1202. [http://dx.doi.org/10.1039/B821856K]

Beier BD, Quivey RG, Berger AJ (2010) Identification of different bacterial species in biofilms using confocal raman microscopy. J Biomed Opt 15(6): 66001
Caspers PJ, Lucassen GW, Bruining HA, Puppels GJ (2000) Automated depth-scanning confocal raman microspectrometer for rapid in vivo determination of water concentration profiles in human skin. J Raman Spectrosc 31(8-9): 813-818

Ellis DI, Goodacre R (2006) Metabolic fingerprinting in disease diagnosis biomedical applications of infrared and raman spectroscopy. Analyst 131: 875-885

Hanlon EB, Manoharan R, Koo TW, Shafer KE, Motz JT, Fitzmaurice M, Kramer JR, Itzkan I, Dasari RR, Feld MS (2000) Prospects for in vivo raman spectroscopy. Phys Med Biol 45(2): R1-R59

Loesche WJ (1986) Role of streptococcus mutans in human dental decay. Microbiol Rev 50(4): 353-380

Maquelin K, Kirschner C, Choo-Smith LP, van den Braak N, Endtz HP, Naumann D, Puppels GJ (2002) Identification of medically relevant microorganisms by vibrational spectroscopy. J Microbiol Methods 51: 255-271

Marsh PD (1999) Microbiologic aspects of dental plaque and dental caries. Dent Clinics North Am 43(4): 599-614

Rösch P, Harz M, Schmitt M, Peschke KD, Ronneberger O, Burkhardt H, Motzkus HW, Lankers M, Hofer S, Thiele H, Popp J (2005) Chemotaxonomic identification of single bacteria by micro-raman spectroscopy: Application to clean-room-relevant biological contaminations. Appl Environ Microbiol 71(3): 1626-1637

Socransky SS, Manganiello SD (1971) The oral microbiota of man from birth to senility. J Periodontology 42(8): 485-496

Wagner M, Ivleva NP, Haisch C, Niessner R, Horn H (2009) Combined use of confocal laser scanning microscopy (CLSM) and raman microscopy (RM) Investigations on EPS matrix. Water Res 43: 63-76

Willemse-Erix DFM, Scholtes-Timmerman MJ, Jachtenberg JW, van Leeuwen WB, Horst-Kreft D, Schut TCB, Deurenberg RH, Puppels GJ, van Belkum A, Vos MC, Maquelin K (2009) Optical fingerprinting in bacterial epidemiology: Raman spectroscopy as a real-time typing method. J Clin Microbiol 47(3): 652-659

doi:10.1186/2191-0855-2-35

Cite this article as: Beier et al:: Raman microspectroscopy for species identification and mapping within bacterial biofilms. AMB Express 2012 2:35.

\section{Submit your manuscript to a SpringerOpen ${ }^{\circ}$ journal and benefit from:}

- Convenient online submission

- Rigorous peer review

- Immediate publication on acceptance

- Open access: articles freely available online

- High visibility within the field

Retaining the copyright to your article

Submit your next manuscript at $>$ springeropen.com 